\title{
Management of mitral regurgitation in Marfan syndrome: Outcomes of valve repair versus replacement and comparison with myxomatous mitral valve disease
}

\author{
Meghana R. K. Helder, MD, ${ }^{a}$ Hartzell V. Schaff, MD, ${ }^{a}$ Joseph A. Dearani, MD, ${ }^{a}$ Zhuo Li, MS, ${ }^{b}$ \\ John M. Stulak, MD, ${ }^{a}$ Rakesh M. Suri, MD, DPhil, ${ }^{a}$ and Heidi M. Connolly, MD ${ }^{c}$
}

\begin{abstract}
Objectives: The study objective was to evaluate patients with Marfan syndrome and mitral valve regurgitation undergoing valve repair or replacement and to compare them with patients undergoing repair for myxomatous mitral valve disease.
\end{abstract}

\begin{abstract}
Methods: We reviewed the medical records of consecutive patients with Marfan syndrome treated surgically between March 17, 1960, and September 12, 2011, for mitral regurgitation and performed a subanalysis of those with repairs compared with case-matched patients with myxomatous mitral valve disease who had repairs (March 14, 1995, to July 5, 2013).

Results: Of 61 consecutive patients, 40 underwent mitral repair and 21 underwent mitral replacement (mean [standard deviation] age, 40 [18] vs 31 [19] years; $P=.09$ ). Concomitant aortic surgery was performed to a similar extent (repair, 45\% [18/40] vs replacement, $43 \%$ [9/21]; $P=.87$ ). Ten-year survival was significantly better in patients with Marfan syndrome with mitral repair than in those with replacement $(80 \%$ vs $41 \%$; $P=.01$ ). Mitral reintervention did not differ between mitral repair and replacement (cumulative risk of reoperation, $27 \%$ vs $15 \% ; P=.64$ ). In the matched cohort, 10 -year survival after repair was similar for patients with Marfan syndrome and myxomatous mitral disease ( $84 \%$ vs $78 \% ; P=.63)$, as was cumulative risk of reoperation $(17 \%$ vs $12 \% ; P=.61)$.
\end{abstract}

Conclusions: Patients with Marfan syndrome and mitral regurgitation have better survival with repair than with replacement. Survival and risk of reoperation for patients with Marfan syndrome were similar to those for patients with myxomatous mitral disease. These results support the use of mitral valve repair in patients with Marfan syndrome and moderate or more mitral regurgitation, including those having composite replacement of the aortic root. (J Thorac Cardiovasc Surg 2014;148:1020-4)

Mitral valve (MV) repair has become the preferred method of surgical treatment for mitral regurgitation (MR). Compared with replacement, valve repair provides better postoperative outcomes, including better long-term survival. ${ }^{1}$ This shift in surgical practice for MV disease has not been as enthusiastically embraced in patients with Marfan syndrome (MFS), in part because of an incomplete mechanistic understanding of the cause of MV prolapse and resulting MR in this population. ${ }^{2}$ Also, many patients with MFS who undergo operations for MR have mechanical aortic valve prostheses and require

From the Divisions of Cardiovascular Surgery, ${ }^{\mathrm{a}}$ Biomedical Statistics and Informatics, ${ }^{\mathrm{b}}$ and Cardiovascular Diseases, ${ }^{\mathrm{c}}$ Mayo Clinic, Rochester, Minn.

Disclosures: Rakesh M. Suri is the Principal Investigator of the PERCEVAL Trial with patent applications and is the Co-principal Investigator of the COAPT Trial (Abbott), and receives grant support from St. Jude, Sorin, and Edwards. All other authors have nothing to disclose with regard to commercial support.

Read at the 94th Annual Meeting of The American Association for Thoracic Surgery, Toronto, Ontario, Canada, April 26-30, 2014.

Received for publication March 27, 2014; revisions received June 16, 2014; accepted for publication June 28, 2014

Address for reprints: Hartzell V. Schaff, MD, Division of Cardiovascular Surgery, Mayo Clinic, 200 First St SW, Rochester, MN 55905 (E-mail: schaff.hartzell@ mayo.edu).

$0022-5223 / \$ 36.00$

Copyright (c) 2014 by The American Association for Thoracic Surgery

http://dx.doi.org/10.1016/j.jtcvs.2014.06.046 anticoagulation with warfarin; thus, clinicians may prefer mechanical MV replacement in an attempt to reduce the risk of future reoperations.

Previous smaller case series suggest that MV repair is durable in patients with $\mathrm{MFS}^{3,4}$; however, durability of repair remains a concern because of the difference in MV morphology compared with that in idiopathic myxomatous MV disease. ${ }^{5}$ Thus, outcomes of MV repair in patients with MFS should be interpreted in light of outcomes of valve replacement and compared with those of patients having repair for myxomatous MV disease. Our hypothesis is that MV repair is superior to MV replacement in patients with MFS with moderate or greater MV regurgitation. Also, outcomes of MV repair in patients with MFS should be comparable to those of MV repair in patients with idiopathic myxomatous MV disease.

\section{METHODS}

After obtaining permission from the Mayo Clinic Institutional Review Board, we reviewed the medical records of consecutive patients with MFS who received surgical treatment for MR between March 17, 1960, and September 12, 2011, at our tertiary care academic medical center. Data were retrieved from the Mayo Clinic electronic medical record, our cardiovascular surgical database, and follow-up questionnaires that include 


\section{Abbreviations and Acronyms \\ MFS $=$ Marfan syndrome \\ $\mathrm{MR}=$ mitral regurgitation \\ $\mathrm{MV}=$ mitral valve \\ $\mathrm{TGF}=$ transforming growth factor}

quality of life measures at $1,3,5,10,15$, and 20 years after surgery. All patients had signed informed consent forms releasing their medical records for use in research studies.

All patients undergoing first-time mitral intervention were analyzed, including those with concomitant aortic surgery. In a subgroup casematched analysis, we compared patients with MFS who underwent MV repair (March 14, 1995, to July 05, 2013) with patients undergoing MV repair for idiopathic myxomatous MV disease. Patients with MFS were case-matched $1: 2$ by sex, ejection fraction $( \pm 5 \%)$, age ( \pm 5 years), and date of operation ( \pm 5 years).

\section{Statistical Analysis}

Descriptive statistics are reported, as appropriate, as number (percentage) for categoric variables and as mean (standard deviation) or median (range) for continuous variables. Categoric variables were compared between the repair group and the replacement group and between matched repair groups using the chi-square test or Fisher exact test, and continuous variables were compared using the 2-sample $t$ test or the Wilcoxon ranksum test, as appropriate. The Kaplan-Meier method was used to estimate survival and rates of freedom from reintervention. All statistical tests were 2 -sided, with an alpha level of .05 indicating statistical significance.

\section{RESULTS}

\section{Entire Patient Cohort}

A total of 61 consecutive patients with MFS underwent surgical treatment for MR during the study period. Forty patients underwent MV repair, and 21 patients underwent MV replacement. Case-matching was possible for 32 patients with MFS, yielding a total of 64 control patients.

Presenting symptoms for the 61 patients with MFS with MR varied; $85 \%(\mathrm{n}=51)$ had dyspnea, $8 \%(\mathrm{n}=5)$ had fatigue, $3 \%(\mathrm{n}=2)$ had palpitations, $3 \%(\mathrm{n}=2)$ had angina, and $1 \%(\mathrm{n}=1)$ had cardiac arrest. The mean age of the 61 patients was 37 (9) years; patients who underwent MV repair were slightly older than those who underwent MV replacement (mean age, 40 [18] vs 31 [19] years; $P=.09$ ). Other demographic characteristics were similar between the MV repair and replacement groups (Table 1). Of the 13 patients undergoing MV repair and concomitant aortic surgery, 6 had aortic valve-sparing root replacements, 1 had an arch replacement, and the rest $(\mathrm{n}=6)$ had aortic valve replacement and repair of the aorta. Of the 9 patients who underwent MV replacement and concomitant aortic surgery, none had valve-sparing aortic root replacement. Most patients in the repair group and the replacement group had MR grades greater than 2 (95\% in both groups [38/40] and [20/21], respectively). The percentage of patients receiving anticoagulation before operation was similar between both groups $(20 \%$
TABLE 1. Characteristics of 61 patients with Marfan syndrome undergoing mitral valve surgery

\begin{tabular}{|c|c|c|c|}
\hline & $\begin{array}{c}\text { MV repair } \\
(\mathbf{n}=\mathbf{4 0})\end{array}$ & $\begin{array}{l}\text { MV replacement } \\
\quad(\mathbf{n}=\mathbf{2 1})\end{array}$ & $P$ value \\
\hline Age, mean (SD), y & $40(18)$ & $31(19)$ & .09 \\
\hline $\mathrm{EF}$, mean $(\mathrm{SD}), \%$ & $60(7)$ & $56(8)$ & .10 \\
\hline Male sex, no. (\%) & $27(68)$ & $14(67)$ & .95 \\
\hline $\begin{array}{l}\text { Preoperative NYHA } \\
\text { class III or IV, no. }(\%)\end{array}$ & $39(98)$ & $19(90)$ & .40 \\
\hline $\begin{array}{l}\text { Concomitant aortic } \\
\text { surgery, no. }(\%)\end{array}$ & $18(45)$ & $9(43)$ & .87 \\
\hline
\end{tabular}
standard deviation.

[8/40] vs $29 \%$ [6/21], respectively). Indications for anticoagulation were atrial fibrillation or prior mechanical aortic valve replacement.

Annuloplasty (78\% [29/37] posterior; $22 \%$ [8/37] complete) was used in most MV repairs (92\% [37/40]). Other repair techniques included triangular or quadrangular leaflet resection of the posterior leaflet (38\% [15/40]), leaflet plication $(15 \%$ [6/40]), neochordae insertion (15\% [6/40]), commissuroplasty $(7.5 \%$ [3/40]), and Alfieri stitch $(2.5 \%$ [1/ $40])$. Five percent of patients (2/40) had primarily anterior leaflet pathology, $32 \%$ of patients (13/40) had bileaflet prolapse, and $62 \%$ of patients $(25 / 40)$ had primarily posterior leaflet pathology. Mechanical prostheses were used in 20 of the 21 patients who underwent MV replacement. The native MV was completely excised in $38 \%$ of patients $(8 / 21)$.

One early death $(1.6 \%[1 / 61])$ occurred in a patient undergoing isolated MV replacement in 1981. Nonfatal in-hospital morbidity included bleeding that required resternotomy (6.6\% [4/61]), transient ischemic attacks (6.6\% [4/61]), atrial fibrillation (4.9\% [3/61]), and prolonged ventilator support $(1.6 \%$ [1/61]). Complication rates were not significantly different between the repair and replacement groups $(P=.14)$.

The mean duration of follow-up was 7 (5) years after MV repair and 12 (8) years after initial MV replacement $(P=.14)$. Ten-year survival was significantly better in patients with MFS undergoing repair versus replacement $(80 \%$ vs $41 \% ; P=.01)$. In the subgroup of patients who underwent concomitant aortic surgery, 10-year survival was $64 \%$ in patients undergoing repair and $49 \%$ in patients undergoing MV replacement $(P=.27)$ (Figure 1).

In the group with initial MV repair $(\mathrm{n}=40), 8$ patients underwent reoperation with MV replacement. Two of these reoperations occurred in the early experience (1960 and 1966) of Mayo Clinic, and in both cases, initial MV repair was performed with leaflet sutures only. One patient had MV replacement for moderate MV regurgitation at the time of replacement of a calcified aortic valve homograft with severe aortic valve regurgitation. Two of the other 5 patients had newly ruptured chordae, 1 to the anterior leaflet 
A

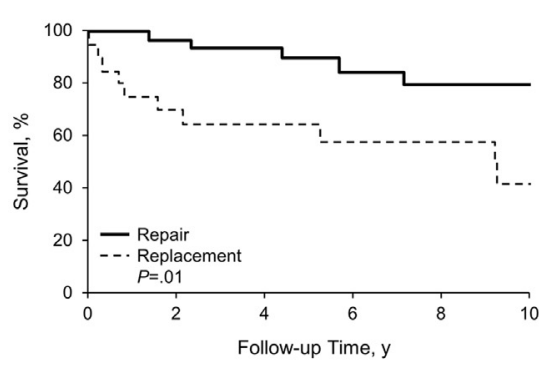

Follow-up Time, y

Repair

B

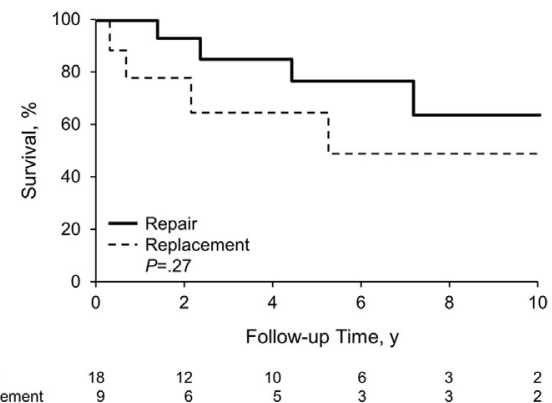

FIGURE 1. A, Overall survival of patients with MFS after MV surgery. Patients with MFS who underwent MV repair had $80 \%$ survival at 10 -year followup compared with $41 \%$ survival for patients with MFS who underwent MV replacement $(P=.01)$. B, Patients with MFS who underwent MV repair with concomitant aortic surgery had a clinically significant better 10-year survival than patients with MFS who underwent MV replacement with concomitant aortic surgery $(64 \%$ vs $49 \% ; P=.27)$.

and 1 to the posterior leaflet. Two of the 5 patients had residual regurgitation without leaflet prolapse, and the cause of the recurrent regurgitation in the fifth patient was indeterminate. Cumulative risk of reoperation 10 years postoperatively was $27 \%$ after MV repair versus $15 \%$ after initial MV replacement $(P=.64)$ (Figure 2$)$.

\section{Matched Patient Cohort}

The most common (77\% [48/62]) presenting symptom in the 62 matched patients with idiopathic myxomatous MV disease was dyspnea. Eight percent (5/62) of the patients presented with fatigue, ventricular tachycardia (secondary to left ventricular hypertrophy), palpitations, or angina (secondary to coronary artery disease). Fifteen percent (9/62) of the patients were asymptomatic. All patients in this group $(\mathrm{n}=62)$ had annuloplasty (81\% [50/62] posterior; $19 \%$ [12/62] complete) as part of the valve repair, and leaflet repair techniques included triangular or quadrangular leaflet resection $(55 \%[34 / 62])$, leaflet plication $(27 \%$

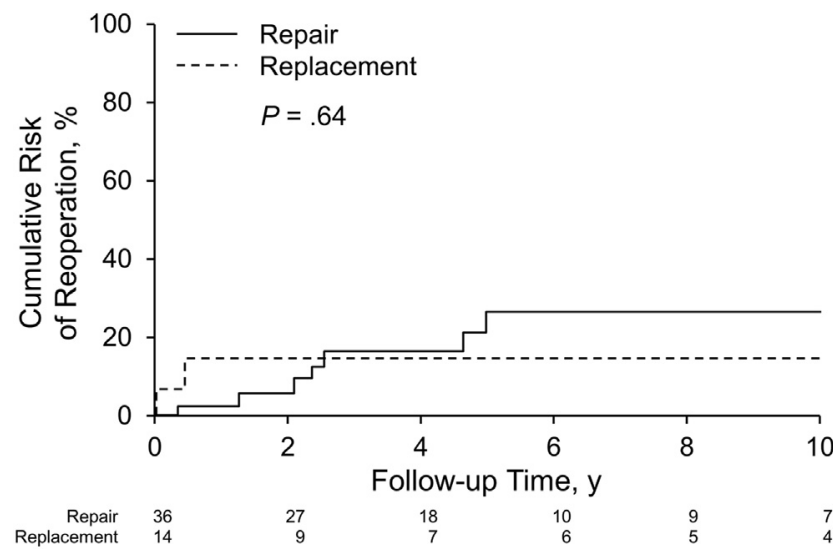

FIGURE 2. Cumulative risk of reoperation of patients with MFS after MV repair or replacement. At 10-year follow-up, patients with MFS who had valve repair had a $27 \%$ chance of mitral reoperation, whereas patients with MFS who had valve replacement had a $15 \%$ chance of mitral reoperation $(P=.64)$.
[17/62]), neochordae insertion (15\% [9/62]), and Alfieri stitch $(5 \%[3 / 62])$.

The mean duration of follow-up was 6.8 (4.5) years in patients with MFS versus 5.8 (4.8) years in patients with idiopathic myxomatous MV disease $(P=.38)$. Survival at 10 years was similar for patients with MFS undergoing MV repair and patients with myxomatous MV disease undergoing MV repair ( $84 \%$ vs $78 \% ; P=.63$ ) (Figure 3$)$. The cumulative risk of reoperation at 10 years was similar for patients with MFS undergoing MV repair and for patients with myxomatous MV disease undergoing MV repair $(17 \%$ vs $12 \% ; P=.61)$ (Figure 4$)$.

\section{DISCUSSION}

The benefit of MV repair over replacement for patients with severe MV regurgitation due to degenerative MV disease is well recognized. An important advantage is low operative mortality. The present study confirms that MV repair or replacement in MFS patients is safe. In our cohort,

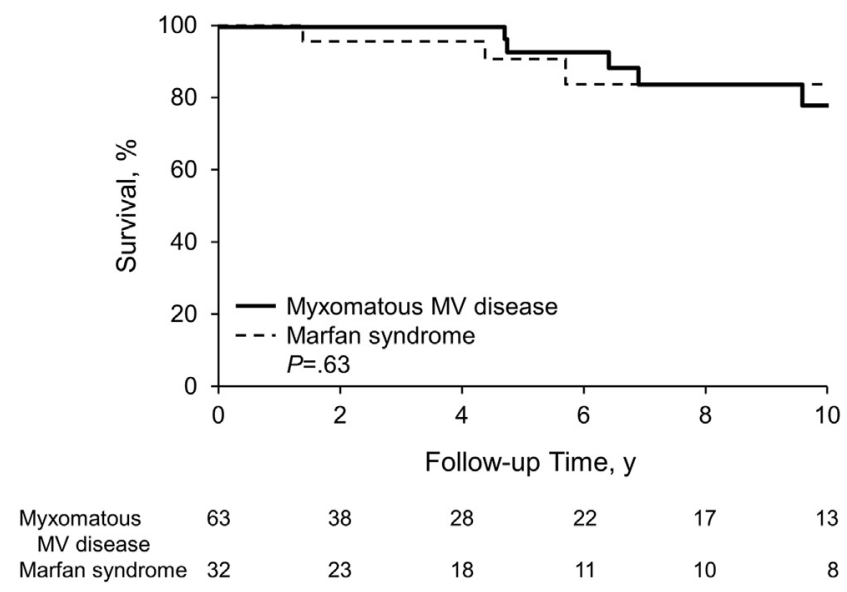

FIGURE 3. Overall survival of patients after MV repair. At 10-year follow-up, patients with MFS who underwent MV repair had an $84 \%$ survival, compared with a $78 \%$ survival for patients with myxomatous MV disease who underwent $\mathrm{MV}$ repair $(P=.63) . M V$, Mitral valve. 


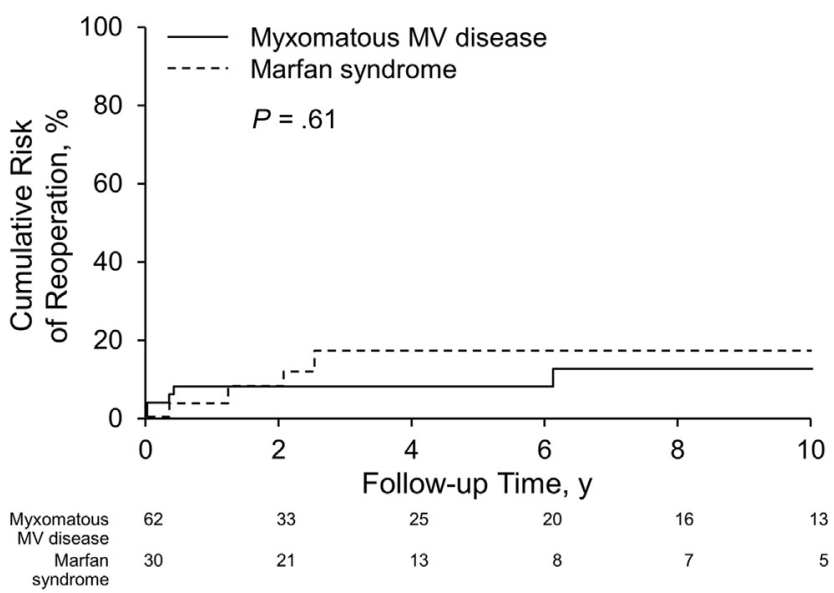

FIGURE 4. Cumulative risk of reoperation of patients after MV repair. At 10-year follow-up, patients with MFS who underwent MV repair had a $17 \%$ chance of mitral reoperation, whereas patients with idiopathic myxomatous MV disease who underwent valve repair had a $12 \%$ chance of mitral reoperation $(P=.61) . M V$, Mitral valve.

the operative mortality was $1.6 \%(1 / 61)$ with no in-hospital deaths since 1981. Other smaller series have reported similar outcomes..$^{3-5}$ This risk of mortality is similar to that for the general population of patients undergoing MV operations. $^{6}$

Compared with prosthetic replacement, MV repair for degenerative valve disease is associated with better preservation of left ventricular function and improved late survival. ${ }^{1}$ Furthermore, for many patients, MV repair avoids the need for long-term anticoagulation with warfarin. However, many patients with MFS and severe MR will require or will have previously undergone composite replacement of the aortic valve and ascending aorta and may require long-term use of warfarin for thromboembolism prophylaxis of the aortic prosthesis. In addition, the potential survival benefit of MV repair for patients with MFS has not been completely established.

In our study, 10-year survival was $80 \%$ in patients with MFS after MV repair and $41 \%$ in patients with MFS after MV replacement. Survival estimates were not adjusted for year of operation, and it is possible that the poorer outcome of patients undergoing valve replacement was related, in part, to the era of surgery. The mechanism of survival benefit may be better preservation of systolic ventricular function, although we could not address specific mechanisms directly in our analysis. However, the difference in late survival does not seem to be related to prosthesisrelated complications, because embolic events were infrequent and did not cause death.

The durability of MV repair has been questioned for patients with MFS or other connective tissue disorders. First, patients with MFS who have MV regurgitation seem to have more frequent involvement of the anterior leaflet than patients with degenerative valve disease. In a study of MV pathology, Bhudia and colleagues ${ }^{5}$ found that patients with MFS had anterior and posterior leaflet prolapse almost as frequently as patients with degenerative MV disease in whom posterior leaflet prolapse predominates. This finding is potentially important regarding durability, because the additional complexity of correction of anterior leaflet prolapse may lead to greater risk of recurrent MR late postoperatively.

In addition, the durability of MV repair in patients with MFS may be compromised because potentially "diseased tissue" remains after repair. But a similar situation exists after repair of myxomatous MVs. Recent histologic studies comparing patients with MFS who have MV disease with those with myxomatous MV disease suggest similarities in valve tissue. Both pathologic processes appear to be associated with fibrillin abnormalities and alteration of the transforming growth factor (TGF)- $\beta$ pathway. The final pathway of MV degeneration in patients with MFS is thought to be mutation of fibrillin-1 protein binding to TGF- $\beta$-activating factors. ${ }^{2}$ Nasuti and colleagues ${ }^{8}$ have identified abnormalities in fibrillin in myxomatous valves as evidenced by more diffuse and weaker staining for fibrillin than that found in normal leaflets. The myxomatous MV with prolapse also has increased TGF- $\beta$ expression. ${ }^{9,10}$ Thus, there may be common pathways of valve degeneration in both MFS and myxomatous MV disease.

In a previous series of patients with MFS undergoing MV surgery, Gillinov and colleagues ${ }^{3}$ reported outcomes of 36 patients (29 repairs, 7 replacements). Only 1 patient required re-repair, necessitated by endocarditis, and no patient required late replacement. Fuzellier and colleagues ${ }^{4}$ reported on 33 patients with MFS who had MV intervention (32 repairs, 1 replacement). Freedom from MV regurgitation was reported as $87 \%$ at 10 years, and no patient had more than 2+ MR during the entire follow-up period. In the present study, the reintervention rate after valve repair in patients with MFS was higher than in previous reports, but our series does include the earliest repairs performed at Mayo Clinic. We found no statistically significant difference in the durability of MV repair when patients with MFS were compared with patients with myxomatous MV disease.

It should be noted that selection of MV repair rather than MV replacement was influenced by other indications for warfarin. Only $15 \%(n=6)$ of our cohort of 40 patients with MFS who underwent MV repair had concomitant or previous aortic valve replacement with a mechanical valve, compared with $62 \%(n=13)$ of the 21 patients with MFS who underwent MV replacement. However, there may be a bias regarding the era of surgery, because many MV replacements were performed in the early experience of Mayo Clinic. A potential advantage for patients undergoing MV repair with a mechanical aortic prosthesis is a lower intensity of anticoagulation with warfarin compared with that required for patients with a mechanical MV prosthesis. 


\section{Study Limitations}

The limitations to this study include its retrospective nature. This cohort is also representative of our singleinstitution, referral-based practice, and thus our findings may not be generalizable. The experience reflects a practice that evolved over 5 decades, although most patients underwent operations in the recent era of MV repair. Finally, although our study examined a large number of patients with MFS who underwent MV surgery, the analysis is underpowered to detect survival differences between the subgroups.

\section{CONCLUSIONS}

To better understand survival and reintervention rates, we case-matched patients with MFS who underwent MV repair to patients with myxomatous MV disease who underwent MV repair; we found no difference in survival or freedom from reoperation. Durability and long-term survival suggest that MV repair in patients with MFS may be preferable to replacement, even in patients who undergo concomitant aortic valve replacement.

\section{References}

1. Enriquez-Sarano M, Schaff HV, Orszulak TA, Tajik AJ, Bailey KR, Frye RL. Valve repair improves the outcome of surgery for mitral regurgitation: a multivariate analysis. Circulation. 1995;91:1022-8.

2. Judge DP, Rouf R, Habashi J, Dietz HC. Mitral valve disease in Marfan syndrome and related disorders. J Cardiovasc Transl Res. 2011;4:741-7.

3. Gillinov AM, Hulyalkar A, Cameron DE, Cho PW, Greene PS, Reitz BA, et al. Mitral valve operation in patients with the Marfan syndrome. J Thorac Cardiovasc Surg. 1994;107:724-31.

4. Fuzellier JF, Chauvaud SM, Fornes P, Berrebi AJ, Lajos PS, Bruneval P, et al. Surgical management of mitral regurgitation associated with Marfan's syndrome. Ann Thorac Surg. 1998;66:68-72.

5. Bhudia SK, Troughton R, Lam BK, Rajeswaran J, Mills WR, Gillinov AM, et al. Mitral valve surgery in the adult Marfan syndrome patient. Ann Thorac Surg. 2006;81:843-8.

6. Rankin JS, He X, O'Brien SM, Jacobs JP, Welke KF, Filardo G, et al. The Society of Thoracic Surgeons risk model for operative mortality after multiple valve surgery. Ann Thorac Surg. 2013;95:1484-90.

7. Suri RM, Schaff HV, Dearani JA, Sundt TM III, Daly RC, Mullany CJ, et al. Survival advantage and improved durability of mitral repair for leaflet prolapse subsets in the current era. Ann Thorac Surg. 2006;82:819-26.

8. Nasuti JF, Zhang PJ, Feldman MD, Pasha T, Khurana JS, Gorman JH III, et al. Fibrillin and other matrix proteins in mitral valve prolapse syndrome. Ann Thorac Surg. 2004;77:532-6.

9. Liu AC, Joag VR, Gotlieb AI. The emerging role of valve interstitial cell phenotypes in regulating heart valve pathobiology. Am J Pathol. 2007;171:1407-18.

10. Barth PJ, Koster H, Moosdorf R. CD34+ fibrocytes in normal mitral valves and myxomatous mitral valve degeneration. Pathol Res Pract. 2005;201:301-4.

\section{Discussion}

Dr Duke Cameron (Baltimore, Md). I have no disclosures. Dr Kunkala, our thanks to you and the Mayo Clinic group for this article, which clearly shows that MV repair has the same low operative risk and good long-term durability as in the patient without MFS with conventional myxomatous MV disease.

My only criticism is that it probably gives an unfairly pessimistic view of the long-term results of MV replacement. Most of your MV replacement cases were still performed in the first half of the series at a time when your patients really didn't have the benefits of modern myocardial protection and postoperative management and our understanding of how the MV works. In $40 \%$ of your replacements, the MV was completely excised, which is something we would rarely do today. I wonder, if you looked at your MV replacements that had been performed in the second half of the series, in a modern era, are the results really as bad as they are with the series overall?

Second, you said in the presentation that most patients with MFS coming from MV surgery are already receiving anticoagulation. I think you probably just meant to say "some." Because certainly in the era in which aortic valve-sparing surgery is becoming so commonplace, the whole idea here is to try to keep them off anticoagulants altogether.

My third comment regards the technique of mitral repair. There is no question that the spectrum of MV disease in MFS is broad, from the simplest $\mathrm{P} 2$ prolapse that you see in myxomatous disease to some of the worst cases of Barlow's that you could imagine.

Our experience with these lesions is that the more complex the mitral pathology, the simpler our repairs have become. You can spend all day transferring cords and resecting leaflets, but some of these extreme valves are best served by a ring, plus or minus the Alfieri stitch, to prevent systolic anterior motion. This has worked well. Can you comment on that strategy?

Dr Helder. Regarding the survival of the patients with MV replacement, you are correct that most of these patients had replacement in an earlier era, and this may have influenced late outcome. But it is important to recognize that valvuloplasty techniques have improved with time, and the outcome of valve repair performed in the earlier era may not be equivalent to results today. Regarding your comment on anticoagulation, you are correct that in current practice many or most patients undergo valve-sparing operations and would not require anticoagulation for an aortic prosthesis. We agree with your strategy for using less complex techniques for MV repair in patients with MFS and for the larger population of patients presenting for MV repair.

Dr Pirooz Eghtesady (St Louis, Mo). I noticed on one of your slides the overall intervention rate for the MV had increased substantially with, obviously, a larger percentage being MV repairs in the most recent era. Is that because indications have changed? You're being more aggressive? How would you characterize that?

Dr Helder. I think the reason for more MV repairs is that surgeons are becoming more comfortable with various repair techniques to address a wider variety of mitral pathologies.

Dr Scott LeMaire (Houston, Tex). Did you look at specific valve-related complications at all, such as thromboembolism or bleeding complications, in the 2 cohorts?

Dr Helder. Of the 21 patients who had MV replacement, 3 developed transient ischemic attacks. So in that small number, I guess we could say that the replacement cases had a little bit more of an embolic phenomenon than the repair cases. Otherwise, we did not see a difference in bleeding or other embolic events in either group because the events were fairly low to begin with.

Dr LeMaire. I may have missed it, but was there any difference in the need for subsequent procedures on the MV in the 2 groups?

Dr Helder. There was not. There was no statistically significant difference between MV reoperation rates between the repair and replacement groups. 